Financial support. Reckitt Benckiser funded the preparation of the manuscript and the figure.

Conflicts of interest. Raymond Nims received a fee from Reckitt Benckiser for writing and editing the manuscript. The other authors report no conflicts of interest relevant to this article.

\section{References}

1. Goldman E. Exaggerated risk of transmission of COVID-19 by fomites. Lancet Infect Dis 2020;20:892-893.

2. Meyerowitz EA, Richterman A, Gandhi RT, et al. Transmission of SARSCoV-2: a review of viral, host, and environmental factors. Ann Int Med 2020. doi: 10.7326/M20-5008.

3. Transmission of SARS-CoV-2: implications for infection prevention precautions. World Health Organization website. https://www.who.int/newsroom/commentaries/detail/transmission-of-sars-cov-2-implications-forinfection-prevention-precautions. Published 2020. Accessed February 8, 2021.

4. Cai J, Sun W, Huang J, et al. Indirect virus transmission in cluster of COVID-19 cases, Wenzhou, China, 2020. Emerg Inf Dis 2020;26:1343-1345.
5. Kanamori H. Rethinking environmental contamination and fomite transmission of SARS-CoV-2 in the healthcare. J Infect 2020. doi: 10.1016/j. jinf.2020.08.041

6. Hirose R, Ikegaya $\mathrm{H}, \mathrm{Naito} \mathrm{Y}$, et al. Survival of severe acute respiratory syndrome coronavirus (SARS-CoV-2) and influenza virus on human skin: importance of hand hygiene in coronavirus disease 2019 (COVID-19). Clin Infect Dis 2020. doi: 10.1093/cid/ciaa1517.

7. Harbourt DE, Haddow AD, Piper AE, et al. Modeling the stability of severe acute respiratory syndrome coronavirus 2 (SARS-CoV-2) on skin, currency, and clothing. PLOS Negl Trop Dis 2020;14(11):e0008831.

8. Ijaz MK, Nims RW, Zhou SS, et al. Microbicidal actives with virucidal efficacy against SARS-CoV-2 and other beta- and alpha-coronaviruses and implications for future emerging coronaviruses and other enveloped viruses. Sci Rep 2021, In press.

9. van Doremalen N, Morris DH, Holbrook MG, et al. Aerosol and surface stability of SARS-CoV-2 as compared with SARS-CoV-1. N Engl J Med 2020;382:1564-1567.

10. Setti L, Passarini F, De Gennaro G, et al. Airborne transmission route of COVID-19: why 2 meters/ 6 feet of inter-personal distance could not be enough. Int J Res Public Health 2020;17(8):2932.

\title{
Could it be that the B.1.1.7 lineage is more deadly?
}

\author{
Chia Siang Kow ${ }^{1}$ and Syed Shahzad Hasan ${ }^{2,3}$ \\ ${ }^{1}$ School of Postgraduate Studies, International Medical University, Kuala Lumpur, Malaysia, ${ }^{2}$ School of Applied Sciences, University of Huddersfield, Huddersfield, \\ United Kingdom and ${ }^{3}$ School of Biomedical Sciences \& Pharmacy, University of Newcastle, Callaghan, Australia
}

To the Editor-The world had been shocked by the emergence of a new variant (B.1.1.7) of severe acute respiratory syndrome coronavirus 2 (SARS-CoV-2), which might have been circulated since September 2020 from the southeastern region of England. ${ }^{1}$ As reported, this new lineage of SARS-CoV-2 has acquired 17 mutations in its genome that lead to amino acid changes within the Spike receptor-binding domain. ${ }^{1}$ The analyses thus far have indicated that the B.1.1.7 lineage might be more transmissible than other SARS-CoV-2 lineages, with a reproduction ratio higher than those of other SARS-CoV-2 lineages by 0.4 and 0.7 (ie, up to $70 \%$ more transmissible). ${ }^{2}$ Santos et $\mathrm{al}^{3}$ employed in silico methods to analyze the interaction between the Spike receptor-binding domain of the B.1.1.7 variant and the ACE2 receptor. They discovered that the N501Y mutant residue on the spike protein of the B.1.1.7 variant establishes a more significant number of interactions with the ACE2 receptor, indicating an increased interaction force with the ACE2 receptor, which could explain its increased infectivity. In contrast, although the newly discovered 501Y.V2 variant, which spread rapidly in the Eastern Cape and Western Cape Provinces of South Africa, also contains the N501Y mutant residue on the spike protein, the substitutions $\mathrm{K} 417 \mathrm{~N}$ and E484K in the South African variant 501.V2 would reduce its binding affinity with ACE2 receptor, resulting in binding affinity comparable to that of the wild-type Spike receptor-binding domain. ${ }^{4}$

Arif ${ }^{5}$ commented that there is uncertainty regarding the severity of disease in people infected with SARS-CoV-2 of the

\footnotetext{
Author for correspondence: Chia Siang Kow, E-mail: chiasiang_93@hotmail.com

Cite this article: Kow CS and Hasan SS. (2022). Could it be that the B.1.1.7 lineage is more deadly?. Infection Control \& Hospital Epidemiology, 43: 678-679, https://doi.org/ 10.1017/ice.2021.59
}

B.1.1.7 lineage. Nevertheless, the general assumption that the B.1.1.7 lineage would not lead to increased severity of COVID19 may not hold true because increased binding affinity between the Spike receptor-binding domain and ACE2 receptor could lead to more ACE2 downregulation should an individual acquire this new variant compared to other variants. ${ }^{6}$ Interestingly, the mutation in the D614G variant, which currently dominates in much of the world, does not increase Spike protein affinity for ACE2.7 Indeed, the $\mathrm{D} 614 \mathrm{G}$ variant is not associated with increased severity of COVID-19 compared to the ancestral strain, although with increased viral load. ${ }^{8,9}$ Until the association between B.1.1.7 lineage and increased severity of COVID-19 is conclusively discredited, perhaps patients who acquire the new B.1.1.7 variant should be managed more aggressively with anti-inflammatory therapies, and the current antiviral armamentarium of COVID-19, especially remdesivir, should be evaluated if it preserves its efficacy against this new variant.

\section{Acknowledgments.}

Financial support. No financial support was provided relevant to this article.

Conflicts of interest. All authors report no conflicts of interest relevant to this article.

\section{References}

1. Kupferschmidt K. Fast-spreading UK virus variant raises alarms. Science 2021;371(6524):9-10.

2. Volz E, Mishra S, Chand M, et al. Transmission of SARS-CoV-2 lineage B.1.1.7 in England: insights from linking epidemiological and genetic data. medRxiv 2020. doi: 10.1101/2020.12.30.20249034. 
3. Santos JC, Passos GA. The high infectivity of SARS-CoV-2 B.1.1.7 is associated with increased interaction force between Spike-ACE2 caused by the viral N501Y mutation. bioRxiv 2020. doi: 10.1101/2020.12.29.424708.

4. Cheng MH, Krieger JM, Kaynak B, Arditi M, Bahar I. Impact of South African 501.V2 variant on SARS-CoV-2 spike infectivity and neutralization: a structure-based computational assessment. bioRxiv 2021. Doi: 10.1101/ 2021.01.10.426143.

5. Arif T. 501.V2 and B.1.1.7 variants of COVID-19: a new time-bomb in the making? Infect Control Hosp Epidemiol 2021. doi: 10.1017/ice.2020.1434.

6. Lei Y, Zhang J, Schiavon CR, et al. SARS-CoV-2 spike protein impairs endothelial function via downregulation of ACE2. bioRxiv 2020. doi: 10.1101/ 2020.12.04.409144.
7. Zhang L, Jackson CB, Mou H, et al. SARS-CoV-2 spike-protein D614G mutation increases virion spike density and infectivity. Nat Commun 2020; 11(1):6013.

8. Groves DC, Rowland-Jones SL, Angyal A. The D614G mutations in the SARS-CoV-2 spike protein: Implications for viral infectivity, disease severity and vaccine design. Biochem Biophys Res Commun 2020. doi: 10.1016/j.bbrc. 2020.10.109.

9. Korber B, Fischer WM, Gnanakaran S, et al. Tracking changes in SARS-CoV2 spike: evidence that D614G increases infectivity of the COVID-19 virus. Cell 2020;182:812-827.

\title{
Warnings regarding the potential coronavirus disease 2019 (COVID-19) transmission risk: Vaccination is not enough
}

\author{
Tianming Zhao MD candidate ${ }^{1, a}(\mathbb{0})$, Chengyang Hu MD ${ }^{1,2, a}$, Mubashir Ayaz Ahmed MD ${ }^{3}$ (1), Ce Cheng DO ${ }^{4}$,, Yue Chen ${ }^{5}$ (1) \\ and Chenyu Sun MD, MSc ${ }^{3}$ (i) \\ ${ }^{1}$ Department of Epidemiology and Health Statistics, School of Public Health, Anhui Medical University, Hefei, Anhui, China, ${ }^{2}$ Department of Humanistic Medicine, \\ School of Humanistic Medicine, Anhui Medical University, Hefei, Anhui, China, ${ }^{3}$ Internal Medicine, AMITA Health Saint Joseph Hospital Chicago, Chicago, Illinois, \\ United States, ${ }^{4}$ The University of Arizona College of Medicine at South Campus, Tucson, Arizona, United States and ${ }^{5}$ Department of Clinical Medicine, School of \\ the First Clinical Medicine, Anhui Medical University, Hefei, Anhui, China
}

To the Editor-Caused by the severe acute respiratory syndrome coronavirus-2 (SARS-CoV-2), the coronavirus disease 2019 (COVID-19) pandemic has continued to spread around the world, resulting in a global health emergency of inconceivable magnitude. ${ }^{1,2}$ Currently, several vaccines, including the PfizerBioNTech COVID-19 vaccine and the Moderna COVID-19 vaccine, have been authorized for emergency use to prevent COVID $-19 .^{3}$ A previous study indicated that the use of a vaccine in combination with measures that reduce contact between susceptible individuals and COVID-19 carriers will significantly decrease the per-day risk of infection as long as at least $50 \%$ of people receive it. ${ }^{4}$ In this article, these researchers also expressed their concern that potential vaccine defiance and abandoning other protection options may cause even worse results in COVID-19 prevention. ${ }^{4}$ In addition, due to the limited supply of COVID-19 vaccine in the United States, Centers for Disease Control and Prevention (CDC) recommends that initial supplies of SARS-CoV-2 vaccine be allocated to healthcare personnel and long-term care facility residents. $^{5}$ Considering the accessibility of vaccines in different regions and populations around the world, public health polices including keeping social distance and wearing face masks, are still of great importance, even though an effective vaccine has been introduced.

Furthermore, according to Morbidity and Mortality Weekly Report from the CDC, ${ }^{6}$ several issues still need to be explored: (1) No data assessing the efficacy of vaccine in prevention of asymptomatic SARS-CoV-2 infection are available; thus, the potential transmission risk of SARS-CoV-2 among asymptomatic

Author for correspondence: Chenyu Sun, E-mail: drsunchenyu@yeah.net aAuthors of equal contribution.

Cite this article: Zhao T, et al. (2022). Warnings regarding the potential coronavirus disease 2019 (COVID-19) transmission risk: Vaccination is not enough. Infection Control \& Hospital Epidemiology, 43: 679-680, https://doi.org/10.1017/ice.2021.63 infected individuals cannot be ignored, even after vaccination. (2) Considering the time interval between the invention of the PfizerBioNTech SARS-CoV-2 vaccine and its emergency use authorization (EUA), the long-term effects of this vaccine (including adverse and protected effects) are still not entirely clear, and further surveillance is still necessary. (3) It takes $\sim 14$ days to obtain protection from infection after the first shot of Pfizer vaccine, ${ }^{7}$ and individuals may still be susceptible during the first few days to weeks after vaccination, whereas the general public may not fully understand this and may be less compliant with current nonpharmaceutical interventions (NPIs) immediately after receiving the vaccine.

In conclusion, uncertainties remain in the long-term effect of SARS-CoV-2 vaccines, and accessibility of vaccines is still limited. Strict public health policies aiming to reduce the spread of SARSCoV-2 are still warranted and should not be ignored.

Financial support. No financial support was provided relevant to this article.

Conflicts of interest. All authors report no conflicts of interest relevant to this article.

\section{References}

1. Twomey JD, Luo S, Dean AQ, et al. COVID-19 update: the race to therapeutic development. Drug Resist Updat 2020;53:100733.

2. Artese A, Svicher V, Costa G, et al. Current status of antivirals and druggable targets of SARS CoV-2 and other human pathogenic coronaviruses. Drug Resist Updat 2020;53:100721.

3. Different COVID-19 vaccines. Centers for Disease Control and Prevention website. https://www.cdc.gov/coronavirus/2019-ncov/vaccines/differentvaccines.html. Published 2020. Accessed February 8, 2021.

4. Abo SMC, Smith SR. Is a COVID-19 vaccine likely to make things worse? Vaccines (Basel) 2020;8(4):E761.

5. When vaccine is limited, who gets vaccinated first? Centers for Disease Control and Prevention website. https://www.cdc.gov/coronavirus/2019- 